\title{
Emergence of Tubulin as a Vaccine against Parasitic Infections
}

\author{
Shreya Rajiv Bhargava and Biswa Prasun Chatterji*
}

Post-Graduate Department of Biotechnology, St. Xavier's College, Mumbai, Maharashtra, India

\begin{abstract}
Tubulin, a conserved cytoskeletal protein, is a well-known drug target. It can also help in combating parasitic ailments. The sequence differences between parasitic and mammalian tubulin have led to the emergence of this protein as a vaccine against the causative agents of the widespread neglected tropical diseases (NTDs). Research on tubulin as a vaccine has resulted in many patented formulations in the past decade. The specific features of these patents (accessed from patent databases-WIPO, Espacenet, US PTO) encompass the source of tubulin, method of production, the vectors and host systems adopted, fusion partners chosen, the purification strategies, incorporation of adjuvants or carriers, route of administration and dosage. The chosen member of the tubulin superfamily for vaccine development is beta tubulin, owing to its variable C-terminus. Most of the patents outline isolation of the desired protein from the parasite; however recombinant and in vitro synthesis of the protein or fragment thereof have also been adopted as viable production systems. Tubulin vaccines reviewed here have been demonstrated as efficacious prophylactic agents against a variety of diseases in animals. These include trypanosomiasis, diseases caused by nematodes, including filariasis, onchocerciasis, and helminthic ailments such as fascioliasis, etc. Optimising additional features such as conformation of the peptide, route of administration, and ascertaining the mechanism of action of the protective antibody would lead to the successful adoption of tubulin vaccines as a promising strategy to ameliorate or eliminate parasitic ailments.
\end{abstract}

Keywords: Tubulin; Drug target; Neglected tropical disease; Vaccine; Trypanosomiasis; Leishmaniasis; Schistosomiasis

\section{Introduction}

Parasitic ailments are a subset of the widespread neglected tropical diseases (NTDs) that affect more than 1 billion people worldwide [1,2]. Of the 17 NTDs outlined by the World Health Organization (WHO), the ones with the highest disease burden and potential for obliteration in the coming decade are visceral leishmaniasis, schistosomiasis, helminth diseases, lymphatic filariasis, onchocerciasis, trypanosomiasis, Chagas' disease etc. [3]. The massive impact of these diseases in rural and economically challenged populations led to the 2000 Millenium Deveopment Goals (MDGs) aiming at their elimination by the year 2015, and more recently, the milestone resolution undertaken by WHO in May 2013 [4,5]. The prevalent methods to control and eliminate these diseases include mass drug administration, or chemotherapy [1]. However, development of drug resistance impedes the efficiency and ability of such chemotherapeutic measures to decrease disease burden at a desired rate [6,7]. Towards meeting this end, national level health policies and support groups have emphasised the need to devise new combating strategies envisaging vector control, vaccine development, improved diagnostics, etc. [1,3,6,8,9].

The past decade has witnessed the evolution of vaccination as a preventive measure in controlling parasitic infections. Parasitic infections have been shown to bestow immunity on ensuing infections by the same parasite in the host, thus authenticating the plausibility of an effective vaccine strategy [10-12]. One of the first commercially developed parasitic vaccines was "Dictol", which functioned against the bovine lungworm Dictyocaulus viviparus and consisted of irradiated larvae of the parasite (Patent number EP 0178928) [13]. More recently, vaccines constituting the excretory or secretory proteins (ES) of the parasites have been developed [14]. Patented vaccine formulations against schistosomiasis, an inflammatory disease caused by the trematode Schistosoma [15], include an integral membrane protein as an antigen [16], paramyosin protein [17], a functionally important antigen Sm-p80 [18], etc. Similarly patents also exist for vaccines against onchocerciasis, caused by Onchocerca [19-21]; leishmaniasis caused by the protozoan parasite Leishmania [22-25]; malaria caused by parasites belonging to the Plasmodium species [26-28]; and vaccines against Trypanosoma, which causes sleeping sickness and Chagas' disease [29-32].

Despite the scale of research, parasite vaccines, whether as whole cells or subunit vaccines, have largely been unsuccessful [10]. The chief reasons for this are the chronic nature of parasites combined with large genetic diversity, inappropriate and ineffective elicitation of immune responses, immune evasion strategies and the complex lifecycles of these parasites, to name a few $[10,14]$. However, insight into structurally and functionally conserved proteins, such as tubulin, [33] has led to their extensive evaluation as putative vaccine targets, as well as their successful incorporation into viable vaccine formulations.

This review aims at outlining the principle features of tubulin as a vaccine target based on patents (accessed from patent databasesWIPO, Espacenet, US PTO) and speculating into the expediency of adopting tubulin vaccines. Towards this end, it is crucial to comprehend the feasibility of tubulin as an immunoprophylactic agent in parasite infections.

\section{Tubulin as a Plausible Target for Vaccine Development- Rationale}

Tubulin is a eukaryotic protein, forming the basic unit of microtubules. Microtubules are hollow, tubular structures constituting

*Corresponding author: Dr Biswa Prasun Chatterji, Assistant Professor PG Department of Biotechnology, St Xavier's College, Mumbai, India, Tel: 02222620661; E-mail: biswaprasun@gmail.com

Received June 11, 2014; Accepted September 02, 2014; Published September 08, 2014

Citation: Bhargava SR, Chatterji BP (2014) Emergence of Tubulin as a Vaccine against Parasitic Infections. Intel Prop Rights 2: 124. doi:10.4172/23754516.1000124

Copyright: (C) 2014 Bhargava SR, et al. This is an open-access article distributed under the terms of the Creative Commons Attribution License, which permits unrestricted use, distribution, and reproduction in any medium, provided the original author and source are credited. 
an essential part of the cytoskeleton [12,34]. The main components of this structural protein are alpha and beta tubulin, which exist in multiple isoforms in different organisms and different tissues. They associate longitudinally as functional dimers to form protofilaments of the microtubule [33,34]. Microtubules constitute the ubiquitous tubular network of spindle fibres that are a part of the cytoskeleton and perform an important function in cell division by making mitotic spindle [12]. Besides, they are also implicated in determination of cell shape and polarity, cellular movements and intracellular transport, maintenance of structural integrity of cellular organelles; all of which are crucial determinants of cell survival [12,35-37].

Parasitic microtubules are spatially and functionally distributed. They include nuclear spindle fibres that form the mitotic apparatus implicated in chromosome segregation, subpellicular microtubules which are responsible for cellular morphology, axonemal tubulin conferring locomotive abilities on the parasite, and the microtubules that constitute an intracellular scaffold for the transport of a wide variety of molecular cargo [38-41]. In kinetoplastid parasites, like those belonging to the Trypanosoma species, there occurs a change in cell morphology and motility when the parasite transits between the vector and host; the basis for this change is the intracellular modifications occurring in the cytoskeleton [42-44]. Even in the intracellular, obligate parasites belonging to Apicomplexa phylum, microtubules facilitate host cell invasion via the conoid, and assist gliding motility and cell division, which are critical attributes in parasite infiltration of the host cells. The subpellicular microtubules impart additional stability to the parasite [45-47]. Thus, as is evident, tubulin is a structurally and functionally decisive protein in the infective, infiltrative and replicative operations of the parasite, making it a viable target for therapeutic intervention.

The suitability of parasitic tubulin as a drug target is augmented by its inherent disparities with mammalian tubulin $[48,49]$. Although tubulin protein is highly conserved, subtle alterations in sequence and structure have been demonstrated [50]. These are due to a number of factors such as, sequence variations in the C-termini of alpha and beta tubulin [50,51], sequence-dependent differential association of the microtubule-associated proteins (MAPs) with tubulin [50], and heterogeneity of post-translational modifications [52].

The sequence variations in the C-termini of tubulin subunits within Homo sapiens and parasites are represented in Figures 1 and 2, deduced by multiple sequence alignment using ClustalW2 software [53]. As is shown in Figure 1a, alpha tubulin of Trypanosoma cruzi shares the least similarity with Homo sapiens, which can be seen to be localised in the C-termini (Figure 1b). Similarly, beta tubulin sequences of T. cruzi and $H$. sapiens are least identical, and the differences are indicated to be confined to the C-termini (Figure 2b).

Collectively, susceptibility of tubulin as a drug target, has given way to the emergence of an armamentarium of anti-mitotic drugs specific for tubulin. These drugs, depending on their preferred binding site, are classified into colchicine analogues, vinca alkaloids and taxanes [54]. Benzimidazoles are a class of drugs that are widely used to treat many protozoan and helminthic ailments [48]. These preferentially bind close to the colchicine binding site buried in the C-terminal beta tubulin [55]. Diversity in amino acid residues constituting the colchicinebinding site in beta tubulin around position 316 is noteworthy. The Leishmania beta tubulin sequence contains ASAL spanning the region 313-316, whereas the mammalian tubulin is composed of the VAAV/I motif [48]. Another class of drugs specifically binding to parasite tubulin subunits is the dinitroanilines (oryzalin and trifluralin) [56,57]. The specificity and high affinity binding of these drugs has been demonstrated by Chan, et al. where trifluralin binds to leishmanial tubulin, while having no effect on rat tubulin [58]. The mechanisms by which the benzimidazole and dinitroaniline drugs eliminate the parasite from the host are inhibition of egg and larval development as well as intestinal damage $[48,59]$. The major problem associated with most anti-mitotic drugs is the development of drug-resistance. Inherent factors of parasitic tubulin, such as changes in structure, conformation, existence of multigene families, amino-acid substitutions, etc. are

\begin{tabular}{|c|c|c|c|c|}
\hline $\begin{array}{c}\text { Input } \\
\text { Sequence }\end{array}$ & Length & $\begin{array}{c}\text { Query } \\
\text { Sequence }\end{array}$ & Length & Score \\
\hline 1 & 451 & 2 & 425 & 75.06 \\
\hline 1 & 451 & 3 & 451 & 84.70 \\
\hline 1 & 451 & 4 & 448 & 81.03 \\
\hline 2 & 425 & 3 & 451 & 83.76 \\
\hline 2 & 425 & 4 & 448 & 79.29 \\
\hline 3 & 451 & 4 & 448 & 89.73 \\
\hline
\end{tabular}

(b)

\begin{tabular}{|c|c|c|c|}
\hline & 400 & 430 & 440 \\
\hline Sequence 1 & MYAKRAF VHWYVGEQMEEQEF & TEAREDTAALEKDYAEVGCDT & TIMLDEDDEEQEF \\
\hline equernce 4 & MYAKRAF VHWYVGEGMEEQEF & SEAREDLAALEKDYEEVGI DS & $\therefore$ LEGEQEGEEY \\
\hline equence 3 & MYAKRAF VHWYVGEGMEEGEF & SEAREDMAALEKDYEEVGVDS & VIEGEGEEEGEEY \\
\hline & MYSKRAF VHWYVQEQMEEQEF & SEAREEY $\cdots \cdots \cdots \cdots$ & $\ldots \ldots \ldots$ \\
\hline
\end{tabular}

Figure 1: (a): Multiple sequence alignment of alpha tubulin protein sequences using clustalW2 software. Sequence 1: Schistosoma mansoni, sequence 2 : Trypanosoma cruzi, sequence 3: Homo sapiens, sequence 4: Onchocercal volvulus. The percentage of identity between sequence is represented in the column corresponding to score. T.Cruzi displays least similarity with Homo sapiens (83.76\%). (b): c- termini of aligned sequences visualized using jalview tool. 


\begin{tabular}{|c|c|c|c|c|c|c|}
\hline $\operatorname{Seq} A \leqslant$ & Name & Length $*$ & $\mathrm{SeqB} \neq$ & Name & Length $\nRightarrow$ & Score $\Rightarrow$ \\
\hline 1 & gi353232516|emb|CCD79871.1] & 444 & 2 & gi|1220544|gb/AAA91956.1] & 442 & 86.2 \\
\hline 1 & gi353232516/emb/CCD79871.1] & 444 & 3 & gi338695/gb/AAB59507.1| & 444 & 95.72 \\
\hline 1 & gi353232516/emb/CCD79871.1] & 444 & 4 & gi3046907/gb/AAC13549.1| & 448 & 89.86 \\
\hline 2 & gi1220544/gb/AAA91956.1] & 442 & 3 & $g(338695 \mid g b / A A B 59507.1]$ & 444 & 84.16 \\
\hline 2 & gi1220544/gb/AAA91956.1] & 442 & 4 & gi3046907/gb/AAC13549.1] & 448 & 79.64 \\
\hline 3 & gi338695/gb/AAB59507.1] & 444 & 4 & gi3046907/gb/AAC13549.1| & 448 & 88.29 \\
\hline
\end{tabular}

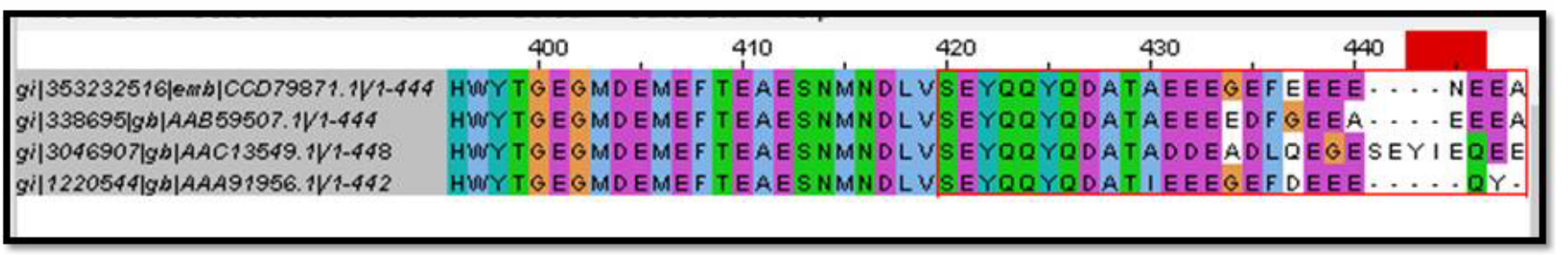

Figure 2: (a): Multiple sequence alignment of beta tubulin protein sequence using ClustalW2 tool. Sequence 1: Schistosoma mansoni, sequence 2: Trypanosoma cruiz, sequence 3: Homo sapiens, sequence 4: Onchocerca volvulus. The percentage of identity between sequence is represented in the column corresponding to score. T.cruiz displays least similarity with homo sapiens (84.16\%). (b): c-termini of algned sequence visualized using jalview tool. Sequences are in the order of S. mansoni, H. sapiens, O. volvulus, T. cruzi.

considered to block drug access [60,61]. Differential expression of microtubule-associated proteins (MAPs) has also been speculated as one of the causes that obstructs drug binding and therefore leads to drug resistance [62]. Emergence of multi-drug resistant parasites has led to therapeutic failure for many parasitic ailments [63]; where majority of the structural alterations leading to resistance have been found to be localised in beta tubulin [61].

These drawbacks of existing chemotherapeutic measures can be circumvented by evaluating tubulin as a vaccine candidate. Consolidated consideration of the properties of tubulin, its consequential role in parasite survival, and its inherent differences from mammalian tubulin has led to the development of tubulin vaccines. The key features of patented vaccines employing tubulin as the antigenic component are analysed below.

\section{Patent Analysis of Vaccines Incorporating Tubulin as the Immunogen}

The major findings that have had an impact in commercial exploitation of tubulin as a vaccine target include, detection of antitubulin antibodies in sera of patients suffering from parasitic infections [64], elucidation of species-specific activity of anti-beta tubulin antibodies from the nematode Brugia pahangi [33], recognition of a tubulin related surface antigen by Trypanosoma cruzi antibodies [65], demonstration of immune-protection against a number of species of Trypanosoma in mice immunised with recombinant beta tubulin from T. evansi [66] and inhibition of trypanosomal growth by anti-beta tubulin antibodies from Trypanosoma brucei [67].

Understanding tubulin as the quintessential candidate for vaccination against a host of parasitic diseases has led to the emergence of a number of patents on the novel production of full-length tubulin proteins or peptide fragments as efficacious vaccines. These are examined below.

WO 94/10201 [68]: This patent discloses one of the first methods employed for tubulin purification and immunisation in Brugiya pahangi. Tubulin isolated from the peritoneal cavity of Gerbils infected with the larvae of nematode Brugia pahangi was found to elicit a specific antibody response in six-week old BALB/c female mice. The spleen cells of the mice were fused with a commercially available myeloma cell line to yield a hybridoma cell line producing monoclonal antibodies against B. pahangi. Of the antibodies thus synthesised, P3D belonging to the IgG isotype recognised an 18 amino acid C-terminal sequence in the beta tubulin of the nematode. This antibody was shown to inhibit the motility and decrease the viability of $B$. pahangi nematodes in culture. The said invention proposes the use of the C-terminal 18 amino acid sequence from beta tubulin of Brugia pahangi as a vaccine to elicit a protective response in the mammal being immunised. The invention also encompasses use of a peptide, synthesised using solid-phase peptide synthesis, and having the same antigenic sequence, as a vaccine. The vaccine can be formulated to include a suitable carrier with or without an adjuvant or immunomodulating agent.

WO 96/32132 [69]: An extension of the above patent, this patent proves the efficacy of the proposed vaccine. In the vaccination-first protocol, gerbils were immunised with $600 \mu \mathrm{g}$ of the beta tubulin peptide over a period of 28 days in 3 phases. They were then infected with 100 larvae of $B$. pahangi two weeks after the last day of immunisation. 36 weeks post infection, the microfilarial burden was estimated and a significant reduction of $95 \%$ was observed. In the infection-first, the gerbils were infected with $100 \mathrm{~B}$. pahangi larvae. 10 weeks following the infection, the peptide vaccine was administered in 3 phases, similar to the vaccination-first protocol. The worm-burden on day 210 was found to be reduced by $85 \%$. The peptide vaccine stimulated antibody production in both, vaccine-first and infection-first protocols. It was equally effective against male and female worms. Additionally, cDNA sequencing of the C-terminal beta tubulin protein from the nematodes Dirofilaria immitis and Onchocerca volvulus reveal sequence conservation amid the beta tubulin of nematodes. This fact is exploited in the patent, where vaccination with beta tubulin from $B$. pahangi is found to confer resistance to infections with $D$. immitis and $O$. volvulus. 
WO 01/58480 [70]: In the said invention, a vaccine rendering protection against Trypanosoma infections in animals and humans is proposed, where immunisation with tubulin from Trypanosoma brucei results in equally effective protection against heterologous strains of $T$. brucei, T. congolense and T. rhodesiense. The sources of tubulin exploited in this invention were purified tubulin from T. brucei, in a native or denatured state and synthetic tubulin peptides (STP). Additionally, rat brain tubulin and rabbit immune sera were also used to characterise the specificity and mechanism of action of the vaccine accurately. Native tubulin was isolated from T. brucei (nTbTub) present in the blood of white rats that had been exposed to the parasite. The tubulin obtained from this strain was subjected to rigorous purification before its use in vaccine studies. The synthetic peptides having sequences corresponding to the carboxy terminal of the beta tubulin were commercially available as fusion proteins with keyhole limpet haemocyanin.

When mice were immunised with a total dose of $80 \mu \mathrm{g}$ of nTbTub, administered in three phases and were subsequently challenged with an optimal dose of T. brucei, $100 \%$ protection was detected. $67 \%$ of these mice were completely protected, while $33 \%$ were partially protected. A comparable or even higher dose of STPs did not protect the mice against Trypanosoma challenge. Studies aimed at determination of effect of dose of nTbTub and adjuvant on protection revealed the minimum effective dose to be $80 \mu \mathrm{g}$. $60 \%$ mice immunised with nTbTub in adjuvant survived beyond 60 days post Trypanosoma challenge, while $0 \%$ survival rate was detected where immunisation was done with nTbTub without adjuvant. The intraperitoneal route of administration of nTbTub resulted in a protection rate of $73 \%$, as against $60 \%$, seen with the subcutaneous route.

Passive transfer of rabbit immune sera led to $80 \%$ protection against challenge, thereby indicating the involvement of a humoral protective response. Immunofluorescence studies in which the intact surface of the trypanosome was stained with antibodies indicated that the antibodies recognise only a small portion of the parasite membrane represented by the flagellar pocket, comprised of axonemal trypanosomes. One of the most important findings by the inventors includes heterologous protection against various strains and species of Trypanosoma on immunisation with the said tubulin antigen. Protection rates of $64 \%$, $73 \%$ and $73 \%$ were seen when mice immunised with nTbTub were challenged with $T$. brucei, $T$. rhodesiense and T. congolense respectively The STPs displayed a weaker degree of antibodyproduction, although heterogeneous activity and specificity for parasite tubulin were detected. The inventors speculate additional features such as conformation as well as composition of the antigen to be responsible for the observations made on STPs.

CA 2378407 [71]: This invention explores various ways in which beta tubulin DNA from the nematodes of the Strongylidae family can be utilised for synthesis of tubulin vaccine. Other applications of the beta tubulin DNA that have been included in the invention are diagnosis of antihelminthic resistance in the nematodes, species identification as well as identification of new antihelminthic or antibiotic compounds.

DNA relating to this invention was in the form of genomic DNA and cDNA. The nucleic acids were recovered from worms of the Strongylidae family, viz. Cyclicocyclus nassatus, Cyathostomum coronatum and Cyathostomum catinatum, which were isolated from the large intestines of infected horses. Similarly, isolation of mRNA and subsequent reverse transcription to cDNA was carried out. The intended beta tubulin was a benzimidazole resistant protein. This would imply a mutation in codon 200 , such that phenylalanine is replaced by tyrosine. Therefore amplification of sequences by means of site-directed mutagenesis using specific primers was carried out. Following this, the DNA was cloned into TA cloning vector. This invention also proposes use of vectors such as $\mathrm{pET}$ vectors or $\mathrm{pGEX}$ vector having appropriate promoters and/or enhancers, functionally linked to the encoding DNA. These vectors also allow for synthesis of histidine (His)-tagged or glutathione-S-transferase (GST)-tagged proteins respectively. This invention highlights use of specific prokaryotic or eukaryotic host systems (strains of E. coli or Baculovirus respectively) to account for maximal synthesis of recombinant protein.

WO 97/47740 [72]: This invention outlines production of peroxiredoxin and/or beta tubulin antigen from cDNA sequences as a vaccine imparting protection against helminth infections. The antigenic material in the form of peroxiredoxin and/or beta tubulin should be as pure as possible, and may comprise of fragments, epitopes, components, precursors, analogues or functional derivatives. The inventors outline a procedure for the individual production of peroxiredoxin and beta tubulin as fusion proteins and evaluate their subsequent formulation into a polyvalent vaccine. The cDNA of either protein species isolated from the liver fluke Fasciola hepatica was engineered to bear an EcoR1 linker arm prior to its incorporation into gt11 lambda phage. This recombinant phage construct was transduced into E. coli cells and the DNA was subsequently isolated before sub-cloning into pGEM-T vector to enable synthesis of beta galactosidase fusion protein. The beta tubulin gene could be synthesised as a fusion protein with Cathepsins L1 and L2, dipeptidyl peptidase or hemoproteins. Incorporation of suitable adjuvants, pharmaceutically acceptable carriers and preservatives are also suggested in the patent. The suggested dose lies in a range of 10-500 $\mu \mathrm{g}$. Cross-protection against other helminths is also an expected feature of this invention.

US 2009/0324634 [73]: This invention relates to a novel method of production of beta tubulin as a vaccine conferring resistance to infestation by the Trypanosoma brucei parasite. The production system encompasses utilisation of recombinant plant expression systems for the synthesis of the different vaccine candidates, namely, full-length alpha and beta tubulin, various fractions of these proteins, microtubuleassociated proteins (MAP15), and composites of alpha and beta tubulin with MAP15 respectively. Plant expression systems bestow many advantages to the production of recombinant proteins, such as post-translational modifications, ease of economically viable largescale production, freedom from animal pathogens, etc. The present invention entails two kinds of fusion partners for the candidate protein antigens, and thereby translates into two kinds of expression system. In the first type, the individual DNA encoding the putative antigens were engineered in RNA-3 vector alongside DNA encoding the Alfalfa Mosaic Virus Coat Protein (CP), which was inoculated onto tobacco (Nicotiana benthamiana) plants. The antigens were expressed as CPfusions trafficked to the viral capsid for expression. Purification of this protein from the leaf was effected using suitable downstream techniques. In the second system, the DNA encoding the target antigens was cloned downstream to a sequence that codes for the thermostable, engineered, Lichenase protein from Clostridium thermocellum. The construct, which also contained endoplasmic or vacuolar retention sequences, was ligated to $\mathrm{pET}$ expression vector. The subsequent infiltration of this vector into N. benthamiana plants, mediated by Agrobacterium species, resulted in the transient expression of the desired protein.

Polytopes of alpha tubulin linked together and bearing a terminal thermostable protein were also included in the study. Mice and cattle were chosen as the model systems for studying the efficacy of the vaccines. In the first immunisation studies on mice, the vaccines were administered 
Citation: Bhargava SR, Chatterji BP (2014) Emergence of Tubulin as a Vaccine against Parasitic Infections. Intel Prop Rights 2: 124. doi:10.4172/23754516.1000124

Page 5 of 7

\begin{tabular}{|c|c|c|c|c|c|c|c|c|}
\hline Patent number & $\begin{array}{l}\text { Antigenic } \\
\text { component }\end{array}$ & $\begin{array}{l}\text { Fusion } \\
\text { partner }\end{array}$ & Recombinant Host & Adjuvant & Target Pathogen & $\begin{array}{c}\text { Animal } \\
\text { Model }\end{array}$ & Dose $(\mu \mathrm{g})$ & $\%$ protection \\
\hline WO 96/32132 & $\begin{array}{l}\text { Beta tubulin } \\
\text { peptide }\end{array}$ & - & - & FCA & Brugia pahangi & Gerbils & 200 & $\begin{array}{l}95 \text { (reduction in } \\
\text { worm burden) }\end{array}$ \\
\hline \multirow{2}{*}{ WO 01/58480 } & Native tubulin & - & - & FCA and IFA & Trypanosoma brucei & Mouse & 80 & 100 \\
\hline & Beta tubulin (STP) & $\mathrm{KLH}$ & - & FCA and IFA & Trypanosoma brucei & Mouse & 200 & 0 \\
\hline US 2009/0324634 & $\begin{array}{c}\text { Alpha tubulin, Beta } \\
\text { tubulin }\end{array}$ & $\begin{array}{l}\text { AlMV CP, } \\
\text { lichenase }\end{array}$ & $\begin{array}{c}\text { Nicotiana } \\
\text { benthamiana }\end{array}$ & $\begin{array}{l}\text { FCA, IFA, } \\
\text { allhydrogel }\end{array}$ & Trypanosoma brucei & Mouse, cattle & 800 & 90 \\
\hline
\end{tabular}

Only the most significant data has been represented. The reader may refer to the patent documents for additional information.

Abbreviations:

FCA - Freund's Complete Adjuvant

IFA - Incomplete Freund's Adjuvant

STP - Synthetic Tubulin Peptide

KLH - Keyhole Limpet Haemocyanin

AIMV - Alfalfa Mosaic Virus

$\mathrm{CP}$ - Coat Protein

Table 1: Essential features of patented tubulin vaccines

in three doses, $400 \mu \mathrm{g}$ (with complete Freund's adjuvant), $200 \mu \mathrm{g}$ (with incomplete Freund's adjuvant) and $200 \mu \mathrm{g}$ (without adjuvant), on day 0 , 14 and 28 respectively. 15 days post the third dose, mice were challenged with $10^{2}$ T. brucei, and parasitemia was monitored. Minimum protection was observed for an alpha tubulin polytope, where $60 \%$ of the mice survived post the challenge. Maximum protection was impacted by beta tubulin, where $90 \%$ of the mice survived. No protection was seen when MAP15 was used individually or as a composite vaccine with alpha or beta tubulin. These studies were coupled with screening of the immune sera of mice for presence of antibodies. ELISA studies revealed the presence of tubulin peptide-specific IgG type of antibodies in the sera, whose values did not correlate with the survival rates observed. The inventors, therefore, inferred on the protection mechanisms to be humoral as well as cell-mediated. Subsequent immunisation studies on mice helped the inventors deduce the beta tubulin peptide conferring maximal protection. Studies on cattle, where different doses of antigen, different adjuvant (allhydrogel), varying count of infective parasite, etc. were used, established no difference between intradermal and intravenous routes of administration. The inventors also speculate the administration of plant-derived vaccines orally, provided the proteins are localised in edible portions of the plant.

\section{Conclusion and Perspectives}

A thorough patent analysis highlights certain fixed principles on which production and utilisation of parasitic tubulin as vaccines are based. The unanimous selection of the C-termini of beta tubulin proteins from the range of parasites underscores its efficacy as a suitable vaccine. Furthermore, as is explicated in all patents, beta tubulin proteins confer cross protection against many species and strains of a chosen parasite. Sequence variations in tubulin found across different organisms are localised in the C-termini of these proteins [54,74], and it is these C-termini that are the most immunogenic in alpha and beta tubulin [74]. Beta tubulin as a highly conserved protein is also an acknowledged feature [75]. Therefore it can be deduced that most sequences incorporated in the vaccines discussed would include the variable portion of the beta tubulin. This would ensure an effective immune response in the host.

Synthesis of the tubulin vaccine as a fusion protein is also noted for some productions. Fusion partners bestow certain advantages like ease of production, purification, and formulation, facilitated incorporation of multiple antigenic determinants, stability of polytopes, and sometimes an enhanced immunogenicity [73]. Most of the inventions showcased the reactivity and efficacy of the monoclonal or polyclonal antibody so produced in elimination of the infective agents. This goes on to justify the existence of a humoral mechanism in amelioration or complete removal of the parasitic agent [73].

Incorporation of adjuvants and carriers in the formulation increases the efficacy of the vaccine [76], and could be the reason that most of the vaccines discussed have appropriate adjuvants and carriers included as an integral part. The patents analysed also underscore the various routes by which the vaccine could be administered, such as subcutaneous, intraperitioneal, sublingual, etc. The degree of prophylaxis conferred by the vaccine would, thus, also be affected by the route of tubulin inoculation in the host (Table 1).

When considering tubulin as a vaccine, an essential facet is the intracellular, cytosolic presence of the protein. Internalisation of the protective antibody into the cytosol of the parasite is a putative mechanism of action [67]. The filarial cuticle is highly absorptive [33]; this could also classify as a reason for uptake of the therapeutic antibody. The integrated presence of tubulin polymers in the plasma membrane, making them more accessible to the external proteins and ligands, may be due to the linking effect of MAPs [67,77]. Plausibility of the antibodies reacting to the membrane-associated microtubules puckered in the flagellar pocket, a deep invagination of the plasma membrane from which the flagella emerges, cannot be ruled out [67]. This flagellar pocket has been shown to take up extracellular ligands by menas of receptor-mediated endocytosis [78]. It is essential to note that these are mechanisms are speculative; a distinct and definite mechanism by which antibodies efficaciously bind to intracellular tubulin yet remains to be established.

Another member of the tubulin superfamily is gamma tubulin, known for its presence in the microtubule-organising centres (MTOCs) and therefore its role in microtubule nucleation $[79,80]$. It is interesting to note that despite being nearly similar to alpha and beta tubulins, its lower abundance and restricted cellular distribution have led to its omission as a vaccine target [77].

Tubulin vaccines reviewed have shown immunoprophylactic effect in trypanosomiasis, diseases caused by nematodes, including filariasis, onchocerciasis, and helminthic ailments such as fascioliasis, etc.

Non-tubulin vaccine targets to cure parasitic diseases, such as paraflagellar rod proteins, trans-sialidase, cysteine, proteinases, acyl carrier proteins,polyproteins, etc. are also in the pipeline [81-84]. However, a major limitation of most of these targets is their stage-specific expression during host transitions of the parasite [73]. These lead to antigenic variations that are distinctive features accompanying parasite development [67]. Efficacy of a vaccine is sure to be compromised if 
Citation: Bhargava SR, Chatterji BP (2014) Emergence of Tubulin as a Vaccine against Parasitic Infections. Intel Prop Rights 2: 124. doi:10.4172/23754516.1000124

such proteins are used as immunoprotective antigens. Tubulin on the other hand is a constitutively expressed protein, that displays fewer biochemical variations during the life-cycle of the parasite $[37,67,85]$.

The protection conferred by tubulin vaccines has a spectrum of applications, not only in mammals, but also in poikilothermic vertebrates like goldfish [86,87]. This highlights their commercial development as an ecologically and economically viable alternative to preventive chemotherapy. However, there are certain factors that must be taken into account to ensure the holistic expansion of the therapeutic potential of tubulin vaccines.

The C-termini of tubulin has highly acidic residues, and a disordered conformation [54]. Therefore optimisation of these structural features so as to derive the most immunogenic conformation in the vaccine formulation is crucial. Another governing factor that can contribute in optimal vaccine formulation is the susceptibility of tubulin C-termini to a range of post-translational modifications [52]. Therefore, tit is obligatory to ensure that the protein or peptide portion of the vaccine retains its molecular conformation post-delivery; thereby highlighting the necessity to validate the route of administration. Structural changes in the C-termini of parasite tubulin could lead to development of vaccine resistance, which could be a major drawback. This aspect needs to be thoroughly investigated to reduce therapeutic failures.

Given the promise of tubulin as a vaccine, speculating its therapeutic efficacy in species of Plasmodium, Schistosoma, Babesia, etc. should be the focal point. Follow-up studies, such as ex vivo testing on mammalian cell lines, are imperative for gaining a holistic understanding of the existing tubulin vaccines and for their commercial development. Moreover, investigating and deducing the accurate mechanism of action by which the protective antibodies access the intracellular tubulin should be the prime future prospect.

Collectively, it would suffice to say that the scientific and commercial exploitation of tubulin vaccines to challenge the risk of mortality and/ or morbidity posed by disease-causing parasites can accelerate the elimination of NTDs, and contribute to the economical, physiological, psychological and sociological well-being of tropical nations.

\section{References}

1. Hotez P, Molyneux D, Fenwick A, Kumaresan J, Sachs S, et al. (2007) Contro of neglected tropical diseases. N Engl J Med 27-357: 1018.

2. Neglected Tropical Diseases (2011)

3. Hotez P (2013) NTDs V.2.0: "Blue Marble Health"-Neglected Tropical Disease control and elimination in a shifting health policy landscape. PLoSNegl Trop Dis 7: e2570.

4. WHO (2014) Millennium Development Goals

5. Smith J, Taylor E (2013) MDGs and NTDs: Reshaping the global health agenda. PLoSNegl Trop Dis 7: e2529.

6. Sangster M, Batterham P, Chapman H, Duraisingh M, Jambre L, et al. (2002) Resistance to antiparasitic drugs: the role of molecular diagnosis. International Journal for Parasitology 32: 637-653.

7. Sibley C, Hunt S (2003) Drug resistance in parasites: can we stay ahead of the evolutionary curve? TRENDS in Parasitology 19: 532-537.

8. WHO (2013) Neglected tropical diseases, The Sixty-sixth World Health Assembly.

9. Hotez P, Bethony J, Bottazzi M, Brooker S, Diemert D, et al. (2006) New technologies for the control of human hookworm infection. TRENDS in Parasitology 22: 327-331

10. Crampton A, Vanniasinkam $T$ (2007) Parasite vaccines: The new generation Infection, Genetics and Evolution 7: 664-673.
11. Vercruysse J, Knox D, Schetters T, Willadsen $P$ (2004) Veterinary parasitic vaccines: pitfalls and future directions. TRENDS in Parasitology 20: 488-492.

12. Dumontet C \& Jordan M.A. (2010) Microtubule-binding agents: a dynamic field of cancer therapeutics Nat Rev Drug Discov. 9(10): 790-803.

13. Urquhart G, Mulligan W, Mcintyre W, Jennings F, Jarret W, et al. (1986) Vaccines. EP 0178928

14. Smith W, Zarlenga D (2006) Developments and hurdles in generating vaccines for controlling helminth parasites of grazing ruminants. Vet Parasitol 139: 347 359.

15. Santos G, Almeida S, Leite L (2006) Schistosomiasis-a century searching for chemotherapeutic drugs. Parasitol Res 99: 505-521.

16. Markovics A, Ram D, Schechter I (1993) Vaccines against schistosomiasis CA 2088078

17. Kurtis J, Jiz M, Wu H, Pond-Tor S (2009) Schistosoma vaccine. WO 2009061407.

18. Siddiqui A, Ahmad G, Zhang W (2011) Schistosomiasis vaccines compositions and methods of use. WO 2011005593.

19. Tripp C, Wisnewski N, Grieve R, Frank G, Richer J (1998) Dirofilaria and onchocerca larval L3 cysteine protease proteins and uses thereof. US 5792624

20. Greene B, Unnasch T (1991) Vaccines. US 5021342

21. Grieve R, Frank G, Wisnewski N (2000) Used to protect animals from diseases caused by parasitic helminths. US 6099843

22. Singh S, Dey A (2010) Constructing a DNA chimera for vaccinedevelopment against leishmaniasis and tuberculosis. WO 20100010577.

23. Bhaumik S, Basu R, Naskar K, Roy S (2007) A hybrid cell vaccine against leishmaniasis [kala-azar]. WO 2007144903.

24. Dutra M, Guimaraes D, Romero O, Tostes G (2009) Recombinant viral vectors and vaccine composition for leishmaniasis. WO 2009089605

25. Fischer L (2010) Canine Leishmania vaccine. 7794736

26. Berzofsky J, Good M, Kumar S, Miller L (1990) Synthetic vaccine against $P$. falciparum malaria. WO 1990000402

27. Kaslow D, Barr P (1998) Transmission-blocking vaccine against malaria. US 5853739.

28. Bigey P, Deloron P, Gnidehou S, Quiviger M, Scherman D, et al. (2012) Vaccines against pregnancy-associated malaria. WO 2012014073.

29. Garg N (2011) Vaccine for control of Trypanosoma cruzi infection and Chagas disease. WO 2011031317.

30. Contreras R, De V, Revelli S (2007) Vaccine against Trypanosoma cruz infection. WO 2007107488.

31. Snary D (1981) Trypanosoma cruzi glycoprotein vaccine for inducing immunity to Chagas' disease. US 4298596

32. Dahlin R, Hungerer D (1982) Chagas vaccine and process for its manufacture killed trypanosome and their use for the manufacture of immunologically active cell constituents. CA1120855.

33. Bughio N, Faubert G, Prichard R (1993) Characterisation and biological activities of anti-Brugia pahangi tubulin monoclonal antibodies. Int $\mathrm{J}$ Parasitol 23: 913-924.

34. Chatterji (2011) Microtubules as antifungal and antiparasitic for drug targets Expert Opin Ther Pat 21: 167-186.

35. Kline-Smith S, Walczak C (2004) Mitotic spindle assembly and chromosome segregation: Refocusing on microtubule dynamics. Mol Cell 15: 317-327.

36. Sato M, Schwartz W, Selden S, Pollard T (1988) Mechanical properties of brain tubulin and microtubules. The Journal of Cell Biology 106: 1205-1211.

37. Robinson D, Sherwin T, Ploubidou A, Byard E, Gull K (1995) Microtubule polarity and dynamics in the control of organelle positioning, segregation, and cytokinesis in the Trypanosome life cycle. The Journal of Cell Biology 128: 1163-1172.

38. Fong D, Lee $B$ (1988) Beta tubulin gene of the parasitic protozoan Leishmania mexicana. Molecular and Biochemical Parasitology 31: 97-106. 
Citation: Bhargava SR, Chatterji BP (2014) Emergence of Tubulin as a Vaccine against Parasitic Infections. Intel Prop Rights 2: 124. doi:10.4172/23754516.1000124

39. Hu K, Roos D, Murray J (2002) A novel polymer of tubulin forms the conoid of Toxoplasma gondii. The Jounal of Cell Biology 156: 1039-1050

40. McKean P, Vaughan S, Gull K (2001) The extended tubulin superfamily. Journa of Cell Science 114: 2723-2733.

41. Rawlings D, Fujioka H, Fried M, Keister D, Aikawa M (1992) Alpha-Tubulin II is a male-specific protein Plasmodium falciparum 56: 239-250

42. Gull K (2001) The biology of kinetoplastid parasites: insights and challenges from genomics and post-genomics. International Journal of Parasitology 31 443-452.

43. Stuart K, Brun R, Croft S, Fairlamb A, Gurtler R, et al. (2008) Kinetoplastids: related protozoan pathogens, different diseases. The Journal of Clinical Investigation 118: 1301-1310.

44. Tyler KM, Luxton G, Applewhite D, Murphy S, Engman D (2005) Responsive microtubule dynamics promote cell invasion by Trypanosoma cruzi. Cellular Mircobiology 7: 1579-1591.

45. Morrissette NS, Sibley L (2002) Cytoskeleton of apicomplexan parasites Microbiology and Molecular Biology Reviews 66: 21-38.

46. Frenal K, Soldati D (2009) Role of the parasite and host cytoskeleton in Apicomplexa parasitism. Cell Host \& Microbe 5: 602-611.

47. Santos JM, Lebrun M, Daher W, Soldati D, Dubremetz J (2009) Apicomplexan cytoskeleton and motors: key regulators in morphogenesis, cell division, transport and motility. International Journal for Parasitology 39: 153-162.

48. Katiyar SK, Gordon V, McLaughlin G, Edlind T (1994) Antiprotozoal activities of benzimidazoles and correlations with $\beta$-tubulin sequence. Antimicrobial Agents and Chemotherapy 38: 2086-2090.

49. Davis C, Gull K (1983) Protofilament number in microtubules in cells of two parasitic nematodes. The Journal of Parasitology 69: 1094-1099.

50. Sullivan KF, Cleveland D (1986) Identification of conserved isotype-defining variable region sequences for four vertebrate $\beta$-tubulin polypeptide classes. ProcNatlAcadSci, USA 63: 4327-4331.

51. Gallo JM, Precigout E (1988) Tubulin expression in trypanosomes. Biology of the Cell 64: 137-143.

52. Hammond J, Cai D, Verhey K (2008) Tubulin modifications and their cellular functions. CurrOpin Cell Biol 20: 71-76.

53. Goujon M, McWilliam H, Li W, Valentin F, Squizzato S, et al. (2010) A new bioinformatics analysis tools framework at EMBL-EBI. Nucleic acids research 38: 695-699.

54. Downing KH (2000) Structural basis for the action of drugs that affect microtubule dynamics. Emerging Therapeutic Targets 4: 219-237.

55. Morgan R, Werbovetz K (2008) Drug targets in kinetoplastid parasites. Landes Biosciences and Springer Science+Business Media.

56. Armson A, Kamau S, Grimm F, Reynoldson J, Best W, et al. (1999) A comparison of the effects of benzimidazoles and the dinitroanilines against Leishmania infantum. Acta Tropica 73: 303-311.

57. Ma C, Tran J, Gu F, Ochoa R, Li C, et al. (2010) Dinitroaniline activity in Toxoplasma gondi expressing wild-type or mutant a-tubulin. Antimicrobial Agents and Chemotherapy 54: 1453-1460.

58. Chang M, Fong D (1990) Inhibition of leishmaniasis but not host macrophages by the antitubulin herbicide trifluralin. Science 249: 924-926.

59. Chatterji B, Jindal S, Srivastava S, Panda D (2011) Microtubules as antifungal and antiparasitic drug targets. Expert Opin Ther Patents 21: 167-186.

60. Lacey $E$ (1998) The role of the cytoskeleton protein, tubulin, in the mode of action and mechanism of drug resistance to benzimidazoles. Int $\mathrm{J}$ Parasito 18: $885-936$.

61. Luis L, Serrano M, Hidalgo M, Mendoza-Leon A (2013) Comparative analyses of the $\beta$-tubulin gene and molecular modelling reveal molecular insight into the colchicines resistance in kinetoplastids organisms. BioMed Research International 2013: 1-8

62. Kavallaris M (2010) Microtubules and resistance to tubulin-binding agents Nature Reviews Cancer 10: 194-204

63. Tian H, Chen B, Wen J (2010) Giardiasis, drug resistance, and new targe discovery. Infect Disord Drug Targets 10: 295-302.
64. Howard MK, Gull K, Miles M (1987) Antibodies to tubulin in patients with parasitic infections. ClinExplmmunol 68: 78-85

65. Alcina A, Fresno M (1988) A tubulin-related 55 kilodalton surface antigen recognized by different Trypanosoma cruzi stage-specific monoclonal antibodies from infected mice. Mol Biochem Parasitol 29: 181-190.

66. Li SQ, Fung M, Reid S, Inoue N, Lun Z (2007) Immunization with recombinant beta-tubulin from Trypanosoma evansi induced protection against T. evansi, T. equiperdum and T. b. brucei infection in mice. Parasite Immunol 29: 191-199.

67. Lubega GW, Ochola D, Prichard R (2002) Trypanosoma brucei: anti-tubulin antibodies specifically inhibit trypanosome growth in culture. Exp Parasitol 102 134-142.

68. Prichard R, Bughio N, Faubert G (1994) Peptides and vaccines derived from nematode tubulin. WO 94/10201.

69. Geary T, Prichard R, Bughio N, Faubert G (1996) Peptides of nematode tubulin and methods of use. WO 96/32132.

70. Prichard R, Lubega G, Byaguraba D, Ochola D (2001) Vaccine against trypanosomiasis. WO 01/58480.

71. Von S, Harder A, Schnieder T, Pape M (2001) DNA coding for beta tubulin and use thereof. CA 2378407

72. Dalton J, Andrews S (1997) Vaccine containing a peroxiredoxin and/or a ß-tubulin. WO 97/47740.

73. Knapp E, Yusibov V (2009) Trypanosome antigens, vaccine compositions, and related methods. US 2009/0324634.

74. de la Vina S, Andreu D, Medrano F, Nieto J, Andreu J (1988) Tubulin structure probed with antibodies to synthetic peptides. Mapping of three major types of limited proteolysis fragments. Biochemistry 27: 5352-5365.

75. Biswas B, Sen K, Choudhury G, Bhattacharyya B (1984) Molecular biology of tubulin: Its interaction with drugs and genomic organisation. J Biosci 6: 431457.

76. Dumonteil E (2009) Vaccine development against Trypanosoma cruzi and Leishmania species in the post-genomic era. Infect Genet Evol 9: 1075-1082.

77. Hemphill A, Seebeck T, Lawson D (1991) The Trypanosoma brucei cytoskeleton: ultrastructure and localization of microtubule associated and spectrin-like proteins using quick-freeze, deep-etch, immunogold electron microscopy. J Struct Biol 107: 211-220.

78. Liu J, Qiao X, Du D, Lee M (2000) Receptor-mediated endocytosis in the procyclic form of Trypanosoma brucei. The Journal of Biochemistry 275: 12032 12040.

79. Scott V, Sherwin T, Gull K (1997) y-Tubulin in trypanosomes: molecula characterisation and localisation to multiple and diverse microtubule organising centres. Journal of Cell Science 110: 157-168.

80. Vayssie L, Vargas M, Weber C, Guillen N (2004) Double-stranded RNA mediates homology-dependant gene silencing of $y$-tubulin in the human parasite Entamoeba histolytica. Molecular \& Biochemical Parasitology 138 $21-28$

81. Dumonteil E, Escobedo-Ortegon J, Rodriguez N, Arjona-Torres A, RamirezSierra M (2004) Immunotherapy of Trypanosoma cruzi infection with DNA vaccines in mice. Infection and Immunity 72: 46-53.

82. Dunning N (2009) Leishmania vaccine: from leishmanisation to the era of DNA technology. Bioscience Horizons 2: 73-82.

83. Gradoni L (2006) Vaccines against leishmanisis: state of the art. Veterinary Research Communications 30: 59-63.

84. Agbo E, Majiwa P, Buscher P, Claassen E, Pas M (2003) Trypanosoma bruce genomics and the challenge of identifying drug and vaccine targets. Trends in Microbiology 11: 322-329.

85. Lubega GW, Byaguraba D, Prichard R (2002) Immunization with a tubulin-rich preparation from Trypanosoma brucei confers broad protection against African trypanosomosis. Experimental Parasitology 102: 9-22.

86. Plouffe D, Belosevic M (2005) Antibodies that recognise $\alpha$ - and $\beta$ - tubulin inhibit in vitro growth of the fish parasite Trypanosoma danilewskyi. Developmental and Comparitive Immunology 30: 685-697.

87. Katzenback BA, Plouffe D, Haddad G, Belosevic M (2008) Administration of recombinant parasite $\beta$-tubulin to goldfish (Carassius auratus L.) confers partial protection against challenge infection with Trypanosoma danilewskyi. Vet Parasitol 151: 36-45 